\title{
Identification of Protein Interaction Partners from Shape Complementarity Molecular Cross-Docking
}

\author{
Elodie Laine ${ }^{1,2}$ and Alessandra Carbone $\mathrm{C}^{1,2}$ \\ 1 Université Pierre et Marie Curie, UMR 7238, Equipe de Génomique Analytique, 15 \\ rue de l'Ecole de Médecine, 75006 Paris, France \\ 2 CNRS, UMR 7238, Laboratoire de Génomique des Microorganismes, \\ Paris 75006, France \\ elodie.laine@upmc.fr, Alessandra.Carbone@lip6.fr
}

\begin{abstract}
There is a growing interest in using efficient shape complementarity docking algorithms to analyze protein-protein interactions at a large scale. We have realized complete cross-docking of several tens of enzyme/inhibitors proteins. On the one hand, we demonstrate that docking score distributions for the known complexes are not distinguishable from those for the non-interacting pairs. On the other hand, we show that the knowledge of the experimental interfaces applied to the docking conformations permits to retrieve true interaction partners with high accuracy. We further identify the determinants of the molecular recognition between true interactors compared to non-interacting proteins.
\end{abstract}

\section{Introduction}

Protein-protein interactions (PPI) regulate virtually all aspects of cell biology. The identification of protein interaction partners is thus of crucial importance to control signaling pathways in physio-pathological contexts. Computational methods such as molecular docking have proven to be valuable tools for the prediction of the native conformations of known protein-protein complexes [24]. Recent studies have also suggested that molecular docking could help retrieve true interacting partners among a set of non-interactors with high accuracy 9 106]. Wass et al. reported that score distributions produced by shape complementarity docking could be used to distinguish 56 known interactors among a diverse background of 922 non-interacting proteins [10. We have shown that the combination of cross-docking simulations and experimental knowledge of protein interfaces successfully identifies known partners within a set of 168 proteins from various functional classes [6]. Our cross-docking simulations employed a refined molecular mechanics energy function and were highly time consuming compared to shape complementarity docking which uses a crude scoring scheme.

In the present work, we investigated whether rigid-body docking using a shape complementarity scoring function only or in combination with experimental knowledge could be used to efficiently identify protein interaction partners

A. Petrosino, L. Maddalena, P. Pala (Eds.): ICIAP 2013 Workshops, LNCS 8158, pp. 318-325, 2013.

(C) Springer-Verlag Berlin Heidelberg 2013 
within a set of 46 enzyme/inhibitors proteins. In the following we report the effectiveness of the two strategies, we discuss the limitations of the docking algorithm we used and the associated scoring function, and we further characterize the conformational ensemble produced by docking.

\section{Results}

\subsection{Docking Score Distributions}

Complete cross-docking (CC-D) was realized on the Enzyme-Inhibitors dataset (46 proteins) of the Mintseris Benchmark 2.0 [7] from unbound conformations. Every one of the 46 unbound structures was docked to all the protein structures in the dataset by the very efficient rigid-body docking algorithm Hex [8]. Each calculation explored about 3 billions candidate ligand-receptor orientations. These were then evaluated and ranked by the Hex scoring function which estimates the shape complementarity between receptor and ligand. The 500 best scores were retained and their statistical distribution was computed. For each protein, the docking score distributions with the known interactor and the other 45 non-interacting proteins were compared using the Wilcoxon rank-sum test [1]. At the $1 \%$ significance level, none of the known true partner's score distributions has significantly better scores than false Enzyme-Inhibitors partners distributions (Table 1, Single column). Less than 10 proteins' known true partners lie within the top $20 \%$ score distributions and slightly more than $50 \%$ of the 46 proteins have their true partner score distribution in the best half of all 46 dataset distributions. We conclude that scores produced by Hex do not carry sufficient information to correctly distinguish known partners from non-interactors within the enzyme-inhibitors dataset considered here.

Table 1. Statistical significance of known true complex docking score distributions

\begin{tabular}{llll}
\hline $\begin{array}{l}\text { Top } \\
\%\end{array}$ & $\begin{array}{l}\text { \# top } \\
\text { proteins }\end{array}$ & \multicolumn{2}{c}{$\begin{array}{c}\text { Enzyme/inhibitors } \\
(\%)\end{array}$} \\
\hline & & Single & Macro \\
\hline 1 & 1 & $0(0)$ & $0(0)$ \\
5 & 2 & $3(7)$ & $1(2)$ \\
10 & 5 & $4(9)$ & $3(7)$ \\
15 & 7 & $6(13)$ & $6(13)$ \\
20 & 9 & $9(20)$ & $7(15)$ \\
30 & 14 & $17(37)$ & $10(22)$ \\
40 & 18 & $21(46)$ & $17(37)$ \\
50 & 23 & $25(54)$ & $24(52)$ \\
\hline
\end{tabular}




\subsection{Using Knowledge of the Interfaces}

As pointed out in previous works 9,6, combining docking scores with the knowledge of the experimental interfaces should help retrieve true interacting partners. This was shown for coarse-grain docking algorithms and we show here that the same holds true for shape complementarity docking. We use a predictive PPI index $(N I I)$ as an estimator of the probability of two proteins to interact. For every protein pair $P_{1} P_{2}$, we determine an optimal interaction index $(I I)$ [6]:

$$
I I_{P_{1}, P_{2}}=\min \left(F I R_{P_{1}, P_{2}} * E_{P_{1}, P_{2}}\right)
$$

where FIR (Fraction of Interface Residues) is the overall fraction of the docking interface composed of residues belonging to the experimentally identified interfaces for the receptor and the ligand: FIR $=F I R_{\text {rec }} * F I R_{\text {lig }}$, and $E$ is the docking score. The minimum is defined over all the docking conformations considered. To compare interaction indexes computed over different pairs, a normalized interaction index, called $N I I$, is computed, that models the symmetric role played by ligand and receptor. $N I I$ is defined in Lopes et al. 6 6 .

The calculation of the NII index for every protein pair of the EnzymeInhibitors dataset enabled to draw a receiver operating curve (ROC) from which known interacting protein pairs could be predicted with an area under the curve (AUC) of 0.81 (Table 2. Single line). This result indicates that the knowledge of the experimental interfaces can be successfully used to evaluate shape complementarity docking conformations and retrieve true interactors. However in practice experimental interfaces are not known and one would wish to replace them by predictions. How precise shall the predictions be to ensure high performance?

Table 2. AUC values for the prediction of known true partners, using complete (all) or partial (S: support, C: core, R: rim) knowledge of the experimental interfaces

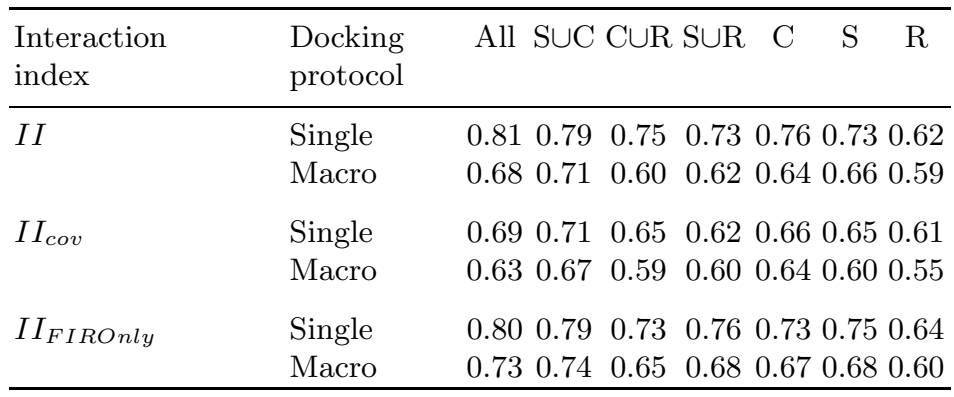

\subsection{Relative Contributions of Interface Sub-regions}

In order to determine whether some interface residues contributed more than others to the discrimination of true partners from non-interactors, we defined 
three sub-regions of the interface depending on residue burial: support, core and rim (Fig. 1). Previous studies highlighted the specific amino acid compositions and the evolutionary conservation profiles of these sub-regions [51]. Using this structural definition of PPI interfaces, we investigated the importance of each sub-region for the prediction of true interacting partners. The AUC value was found similar when discarding information about the rim (0.79 in Table 2). By contrast AUC values significantly decreased down to 0.75 or 0.73 when ignoring support or core in the definition of the experimental interface. Moreover the knowledge of the core alone led to an AUC value of 0.76 as high as that obtained when using both core and rim (0.75). The knowledge of the support alone gave an AUC value of 0.72 similar to that obtained when using both support and rim. The sole knowledge of the rim led to a drastically lower AUC value of only 0.62.

These results show that the core and the support contain more relevant information than the rim for the identification of true interacting partners in the Enzyme-Inhibitors dataset. As a consequence, strategies to predict protein interfaces should concentrate on these two sub-regions. We shall note that this observation may not necessarily hold true for other functional classes of proteins.

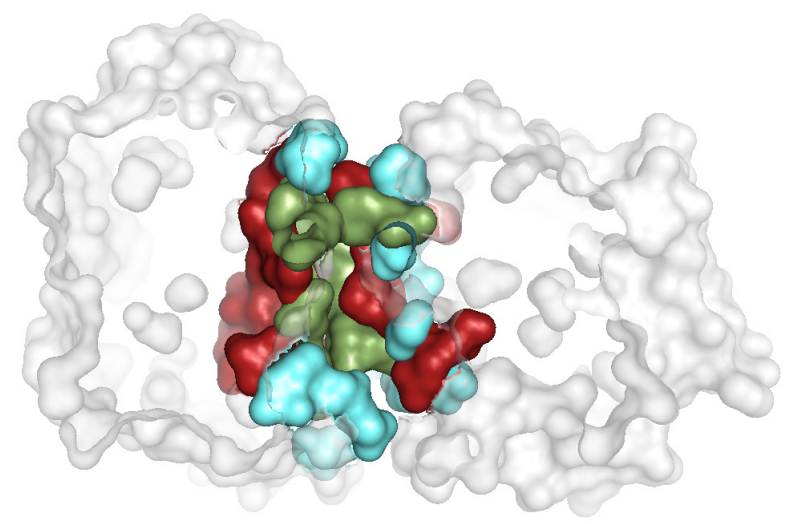

Fig. 1. Example of an enzyme/inhibitor complex (PDB code: 1AVX) from the Mintseris Benchmark 2.0 depicting the three interface sub-regions. The two partners are displayed in transparent light grey surface. The support, core and rim residues are displayed as opaque surface and colored in red, green and cyan respectively.

\subsection{Positive Predictive Value vs Sensitivity}

We also investigated whether it is more important for a docking interface to cover a large part of the experimental interface or be restricted to the residues that truly compose the experimental interface. The FIR computed to determine the interaction index $I I$ represents the overall fraction of the docking interface that is composed of residues belonging to the experimentally identified interface (Eq. 11). This formula is different from that employed in [9], where the fraction 
considered was that of the experimental interface covered by the docking interface, which we refer as $F I R_{\text {cov }}$. Here we also computed the corresponding alternative interaction index $I I_{c o v}$. The AUC values obtained from the calculation of $I I_{c o v}$ are systematically lower than those obtained from $I I$ (Table 2). In particular when considering complete knowledge of the interface, the AUC value drastically decreased down to 0.69 .

These results indicate that to be able to discriminate true from false partners it is more crucial to consider the portion of the docking interface that matches the experimental interface rather than the portion of the experimental interface that is covered by the docking interface. Consequently, methods designed to predict PPI should aim at maximizing positive predictive value rather than sensitivity.

\subsection{Conformational Ensemble Generation and Selection}

In the following we explored the robustness of the docking results and the contribution of the scoring function. To perform CC-D, we used the initial PDB files representing the $3 \mathrm{D}$ coordinates of the 46 proteins studied in unbound forms, superimposed on the bound conformations in the 23 native complexes. In order to investigate whether these initial locations and orientations could bias the results, we performed unbiased CC-D by (i) normalizing the initial distance between receptor and ligand models prior to docking and (ii) generating five initial docking orientations for the ligand over the receptor. The calculation of $I I$ from the obtained conformational ensemble furnished a significantly lower AUC value of 0.68 (Table 2, Macro line). AUC values obtained when using partial knowledge of the experimental interfaces or/and using $I I_{\text {cov }}$ are also systematically lower for the Macro docking compared to the Single docking. This suggests that indeed a favorable bias is introduced towards true known complexes when docking is performed starting from their native conformations.

In order to evaluate the contribution of Hex complementarity score to the identification of true partners, we computed an alternative version of the interaction index $I I_{F I R O n l y}$ that does not incorporate docking scores : II FIROnly $=$ $\max \left(F I R_{P_{1}, P_{2}}\right)$. The AUC values obtained from the calculation of $I I_{F I R O n l y}$ are similar to and sometimes better than those obtained from $I I$ for Single docking and they are systematically better for Macro docking (Table 2). This suggests that the contribution of the docking score is minor in the discrimination of the true complexes from the non-interacting protein pairs and that taking it into account can even deteriorate performance.

\subsection{Characterization of Docking Conformations}

In Fig. 2 we show the distributions of the docking conformations score-based ranks corresponding to three different intervals for the coverage $F I R_{\text {cov }}$ of the support, core and rim. Only conformations whose global FIR is greater than 0.4 are considered. First we observe that docking conformations displaying high coverage of the experimental interface are not ranked first by the scoring function of Hex. Ranks are distributed rather uniformly whatever the value of the $F I R_{c o v}$ 


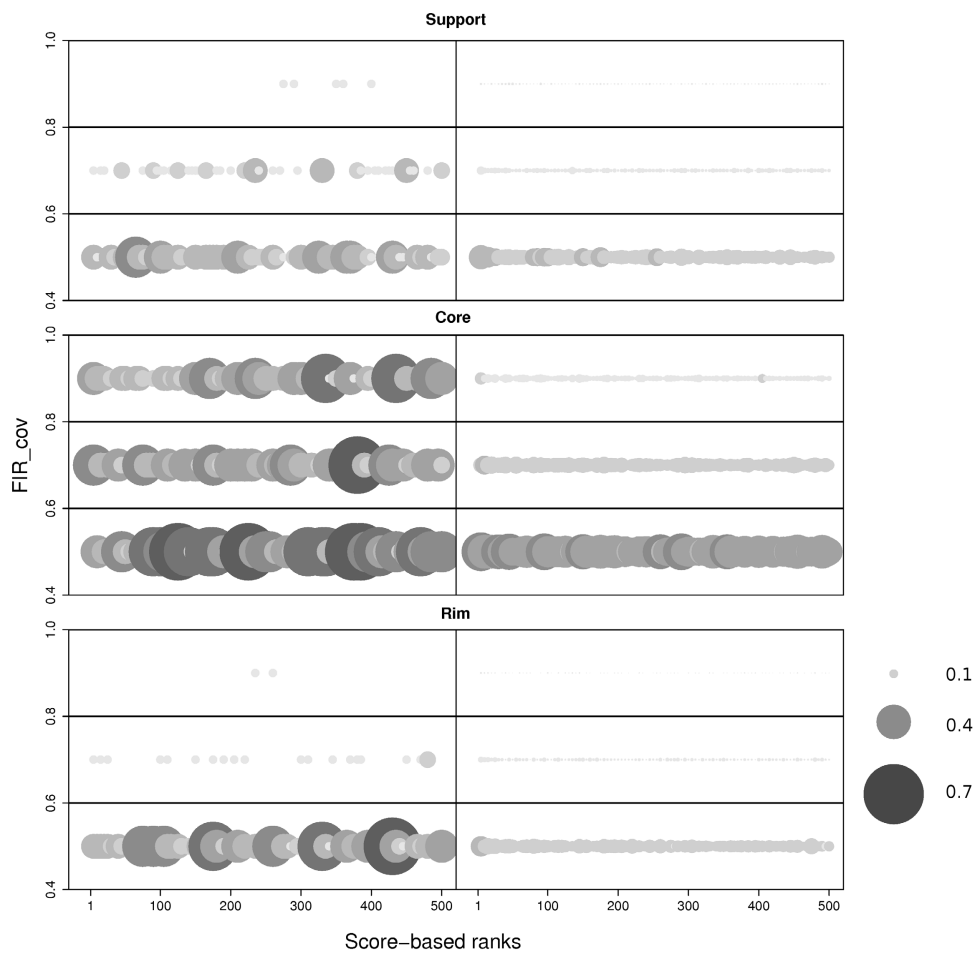

Fig. 2. Comparison of docking conformations for known true complexes (on the left) and non-interacting protein pairs (on the right). Among the 500 best-scored conformations of each docking experiment, those whose $F I R$ is greater than 0.4 are plotted with respect to $F I R_{\text {cov }}$ (y-axis) and score-based ranks (x-axis) for each of the three interface sub-regions: support, core and rim. The y-axis is defined with respect to three main intervals, $[0: 4 ; 0: 6],(0: 6 ; 0: 8]$ and $(0: 8 ; 1: 0]$, and the $\mathrm{x}$-axis varies between 1 and 500 . Each interval on the y-axis is associated to a distribution of ranks, where a bin in the distribution corresponds to 5 consecutive ranks. Bins are represented as circles, their sizes are normalized and vary from 0.1 to $0.7 \%$ of the total number of conformations. Grey levels are redundant with sizes.

and this is true for the three sub-regions. This agrees with the observed poor and even deleterious contribution of the docking score to the identification of true partners. Second, the comparison of docking conformations for known true complexes (Fig. 2 left panels) and for non-interacting protein pairs (Fig. 2, right panels) clearly shows that the coverage of the three sub-regions is greater for the known true complexes. Third, the comparison of the three sub-regions indicates that the coverage of the core region is much larger than those of the rim and support regions. This is in agreement with the central position of the core in the interface. Residues from the rim, which lie at the periphery are the least covered.

Finally, we shall notice that the conformations considered $(F I R>0.4)$ represent a small portion of the total number of docking conformations, respectively 
4.5\% and $3.0 \%$ for the known true complexes and the non-interacting pairs. Similar fractions $(4.1 \%$ and $3.1 \%)$ were computed for Macro docking. This reveals that the large majority of conformations generated and selected by Hex display docking interfaces very different from experimental interfaces.

\section{Discussion}

Our work showed that true interacting partners can be distinguished from noninteractors within a set of 46 enzyme/inhibitors proteins with high accuracy when combining shape complementarity cross-docking and knowledge of the experimental interfaces. This is possible because docking conformations for the known complexes resemble experimental interfaces more that those for noninteracting pairs. In particular, some specific structural sub-regions of the interface largely contribute to the discrimination. The quality of the conformational ensemble generated by the docking algorithm and then selected by the shape complementarity scoring function is an essential element for the performance of our strategy. In a number of cases the true interacting protein pair was not identified as the best match from the docking results but lied in a certain top percentage of candidates. This observation calls for the development of methodologies that account for a confidence level in the determination of potential interacting partners instead of considering only the best one. The method we propose here could be adapted when experimental data of proteins' interfaces are lacking, for example by combining molecular dynamics simulations and ensemble-based docking.

\section{Methods}

Complete Cross-Docking. CC-D was realized on the 46 Enzyme-Inhibitors proteins of the Mintseris Benchmark 2.0 (http://zlab.bu.edu/benchmark2/) from unbound conformations, leading to 2116 docking experiments. Docking was performed with Hex v6.3 [8] using the shape complementarity scoring function. The precision of the molecular representation was defined from 18 and 25 expansion orders for the initial and final search steps. Unbiased CC-D was realized by positioning the receptor and ligand models at a distance of $100 \AA$ from one another prior to docking. Moreover, five starting positions were defined using Hex Macro-Sampling module and were used to generate initial docking orientations for the ligand over the receptor and to derive appropriate local coordinate frames. Among all conformations explored during docking search, the 500 best-scored ones were retained for analysis.

Statistical Testing. Score distributions for the known complexes and the noninteracting protein pairs were compared following a protocol similar to that reported in [10]. For each known true complex, 45 individual one-sided Wilcoxon rank-sum tests [11] were performed, to determine if the ranked set of docking scores from that complex was significantly better (less) than (at P-value $<0.01$ ) the ranked set of docking scores from each of the false complex docking. 
Support-Core-Rim Definition. The three interface sub-regions were defined based on the per-residue relative accessible surface areas (rasa), computed by NACCESS 2.1.1 [3], with a probe size of $1.4 \AA$. For crystallographic structures, interface residues are those displaying any variation $\Delta$ rasa between bound (b) and unbound $(\mathrm{u})$ conformations. The support is composed of residues buried in both conformations rasa $_{u}<0.25$, rasa $\left.b<0.25\right)$. The core contains exposed residues that become buried upon binding to the partner $\left(\right.$ rasa $_{u} \geq 0.25$, $\left.\mathrm{rasa}_{b}<0.25\right)$. The rim is defined by residues exposed in both unbound and bound conformations $\left(\right.$ rasa $_{u} \geq 0.25$ rasa $\left._{b} \geq 0.25\right)$. For docking conformations, residues are considered at the interface when they display a change of at least $10 \%$ decrease in accessible surface area compared to the unbound protein.

Acknowledgements. The MAPPING project (ANR-11-BINF-0003), supported by the Excellence Programme "Investissement d'Avenir", supported this analysis.

\section{References}

1. Andreani, J., Faure, G., Guerois, R.: Versatility and invariance in the evolution of homologous heteromeric interfaces. PLoS Comput. Biol. 8, e1002677 (2012)

2. Gray, J.J.: High-resolution protein-protein docking. Curr. Opin. Struct. Biol. 16, 183-193 (2006)

3. Hubbard, S., Thornton, J.: NACCESS. Tech. rep., Computer Program, Dept of Biochemistry and Molecular Biology, University College London (1993)

4. Janin, J.: Protein-protein docking tested in blind predictions: the CAPRI experiment. Mol. Biosyst. 6, 2351-2362 (2010)

5. Levy, E.D.: A simple definition of structural regions in proteins and its use in analyzing interface evolution. J. Mol. Biol. 403, 660-670 (2010)

6. Lopes, A., Sacquin-Mora, S., Dimitrova, V., Laine, E., Ponty, Y.: Protein-protein interactions in a crowded environment via cross-docking and evolutionary information (submitted, 2013)

7. Mintseris, J., Wiehe, K., Pierce, B., Anderson, R., Chen, R., Janin, J., Weng, Z.: Protein-Protein Docking Benchmark 2.0: An update. Proteins 60, 214-216 (2005)

8. Ritchie, D.W., Kemp, G.J.: Protein docking using spherical polar Fourier correlations. Proteins 39, 178-194 (2000)

9. Sacquin-Mora, S., Carbone, A., Lavery, R.: Identification of protein interaction partners and protein-protein interaction sites. J. Mol. Biol. 382, 1276-1289 (2008)

10. Wass, M.N., Fuentes, G., Pons, C., Pazos, F., Valencia, A.: Towards the prediction of protein interaction partners using physical docking. Mol. Syst. Biol. 7, 469 (2011)

11. Wilcoxon, F.: Individual comparisons by ranking methods. Biometrics Bull. 1, 80-83 (1945) 